\title{
Efficacia retorica e politica delle metafore animalistiche nella predicazione di Savonarola. Il caso della Crocifissione mistica di Botticelli
}

Métaphores animales dans la prédication de Savonarole : efficacité rhétorique et politique. Le cas de la Crucifixion mystique par Botticelli

Animal Metaphors in Savonarola's Preaching: Rhetorical and Political Efficacy.

The Case of Botticelli's Mystical Crucifixion

\section{Marco Versiero}

\section{OpenEdition}

\section{Journals}

\section{Edizione digitale}

URL: http://journals.openedition.org/cei/6074

DOI: $10.4000 /$ cei.6074

ISSN: 2260-779X

\section{Editore}

UGA Éditions/Université Grenoble Alpes

\section{Edizione cartacea}

ISBN: 978-2-37747-159-1

ISSN: 1770-9571

\section{Notizia bibliografica digitale}

Marco Versiero, «Efficacia retorica e politica delle metafore animalistiche nella predicazione di Savonarola. II caso della Crocifissione mistica di Botticelli», Cahiers d'études italiennes [Online], 29 | 2019, online dal 30 septembre 2019, consultato il 27 mars 2021. URL: http://journals.openedition.org/cei/ 6074 ; DOI: https://doi.org/10.4000/cei.6074

Questo documento è stato generato automaticamente il 27 mars 2021. 


\title{
Efficacia retorica e politica delle metafore animalistiche nella predicazione di Savonarola. Il caso della Crocifissione mistica di Botticelli
}

\author{
Métaphores animales dans la prédication de Savonarole : efficacité rhétorique et \\ politique. Le cas de la Crucifixion mystique par Botticelli \\ Animal Metaphors in Savonarola's Preaching: Rhetorical and Political Efficacy. \\ The Case of Botticelli's Mystical Crucifixion
}

Marco Versiero

«Io parlo testé da uomo animale» ${ }^{1}$.

\begin{abstract}
Autorizzato dal gonfaloniere «fratesco» Giuliano Salviati e principiato l'11 febbraio 1498, l'ultimo ciclo predicatoriale di Girolamo Savonarola, dedicato all'Esodo, culminò il 3 marzo col settimo sermone, nel quale i tiepidi (cattivi prelati dal cuore non riscaldato dall'autentica fede) e gli ambiziosi (cattivi cittadini avversi alla riforma repubblicana) erano infine accomunati nella rampogna del frate di San Marco, le cui fortune erano state ormai fatalmente compromesse dal breve di scomunica emesso a suo carico da papa Alessandro VI sin dal giugno dell'anno precedente. Ne conseguiva, agli occhi del ferrarese, una esiziale identificazione della temuta figura di un "tiranno cittadino" con quella, altrettanto bieca, del "tiranno ecclesiastico" (assimilato al faraone egizio): invano, un redivivo Mosè liberatore (Savonarola stesso) si era levato contro quest'ultimo, simile a un cattivo papa quale fu Bonifacio VIII, che al soglio pontificio «entrò come volpe e morì come cane» ${ }^{2}$. La caustica metafora animalistica, intesa nel suddetto sermone a satireggiare una natura astuta e rapace che finisce nondimeno con l'essere addomesticata, costituisce un esempio estremo di ricorso a questo strumento retorico da parte di Girolamo, che di immagini bestiali si era del resto avvalso nel corso della sua intera vicenda politica, servendosene, anzi, per fissare icasticamente alcuni tra i momenti cruciali della sua predicazione fiorentina.
\end{abstract}


2 Reagendo allo statuto ciceroniano che faceva di metafore e allegorie figure stilistiche a mero seducente ornamento del testo, Savonarola attribuiva valore pedagogico alle sue immagini letterarie, non disconoscendone del tutto la ricercatezza estetica ma mirando in ultima istanza al fine della persuasione: «Il predicar a pompa è cosa che solo diletti l'orecchie e non muova il cor dell'uomo»'. Difatti, apprezzandone l'uso (filosofico, non poetico) fattone da Platone (secondo quanto rivendicato nell'Apologeticus de ratione peeticae artis), il domenicano reputa che la metafora, in quanto mezzo simbolico volto a istruire i fedeli, sia chiamata a dirigerne rettamente l'animo all'avvenire, anche illustrandone le possibili e temibili derive.

3 Raramente, nell'infervorata enfasi apocalittica del frate, tramandata dalla foga dei suoi testi, il mondo della natura e delle creature che lo popolano è ritratto come scenario ameno: persino quando, ricorrendo ad efficace travestimento botanico, Savonarola fa di Cristo un «ortolano» intento a coltivare il «giardino» di Firenze, insinua tra i fiori e i frutti degli «arbori grandi» l'insidia di «spine» minacciose (predica $\mathrm{n}^{\circ}$ XXI del 25 dicembre 1494, dal ciclo sopra Aggeo). In questa sorta di versione mondana del giardino dell'Eden, dai connotati fiorentini, appare anche il «serpente che ingannò Madonna Eva», come alter ego bestiale (e biblico) di chi si opponga alla salubre istituzione del Consiglio Grande (predica $\mathrm{n}^{\circ} \mathrm{XVI}$ del ciclo sopra i Salmi, maggio 1495). Del resto, già nella predica dell'Avvento del 1493 ( $\left.\mathrm{n}^{\circ} \mathrm{XXIII}\right)$, l'intervento dell'Onnipotente era stato invocato affinché liberasse la Chiesa «dalle mani de' diavoli, de' tiranni, de' cattivi prelati», orda presto tracimata in una visionaria schiera di fiere: «Non vedi tu che l'è piena d'animali, piena di bestie, leoni, orsi e lupi, che l'hanno tutta guasta?».

4 Il convincimento circa la natura diabolica, folle e bestiale del tiranno si fa apicale nella nona predica nel 25 febbraio 1496 (dal ciclo sopra Amos): «A me pare uno diavolo e peggio ancora», "che la prima cosa e' perde il cervello» ${ }^{4}$. Ne è preludio, d'altronde, la trasposizione allegorica del passato reggimento mediceo, nella predica del 5 luglio 1495 (dal ciclo sopra i Salmi), nella terrifica evocazione di un "governo mostruoso», una sorta di tetramorfo risultante da una abnorme composizione, come per innesto sul corpo del tiranno di membra di tre bestie nefande:

[...] aveva il capo di leone, le spalle e le braccia d'orso, il resto della parte posteriore erano di cane [...]. Il capo di leone significa superbia, perché il leone vuole essere il primo fra gli animali; le braccia d'orso, queste erano le mani a tirare a sé la roba [...]; le posteriori di cane, i satelliti che gli abbaiavano d'intorno [.... $]^{5}$.

5 La fierezza del leone, fattasi superbia peccaminosa, è attributo bestiale del demonio anche nella predica del giorno dell'Ascensione (4 maggio) del 1497, a proposito dei dissidi e dei disordini a suo dire istigati dalla nefasta entità nella platea dei fiorentini raccoltisi ad ascoltarlo: «il demonio, capo di tutti i cattivi [...], va ruggendo come un lione ed eccitando tutti i suoi membri contra me» ${ }^{6}$. Era, il leone di parte guelfa, il Marzocco una cui effigie aveva sostituito a Ponte Vecchio una leggendaria statua sacra al dio della guerra persa con l'alluvione del 1333 (donde Martocus, "piccolo Marte»), il simbolo dell'audace e ruggente fierezza della città. Donatello, nel 1420, ne aveva solennemente sigillato l'icona plastica in Piazza della Signoria (la si ammira oggi in originale al Museo del Bargello), in atto di reggere tra le zampe il blasone con l'insegna dell'iris florentina, contraddistinto dal motto: «Si leo rugiet, quis non timebit?». Eppure, proprio quell'orgoglioso ruggito diviene un verso inquietante e nocivo nella predicazione di Savonarola, che sceglie, non casualmente, di declinare l'immagine 
leonina in via speculare e negativa, proprio a significare la corruttela morale e politica proliferata nella repubblica del giglio.

Ne è eloquente dimostrazione l'articolata metafora animalistica estesamente riversata nella predica, assai nota, del 12 marzo 1497 ( ${ }^{\circ}$ XL del ciclo sopra Ezechiele), della quale converrà nondimeno riportare i principali stralci:

Che vuol dire, populo cristiano? Che la madre tua è leonessa e dorme [...] tra' leoni? [...] Ella è la Chiesa, li preti, li prelati, li principi: mettili tutti insieme. Questi sono la madre, ma principaliter sono quelli che hanno cura delle anime. [...] La donna è diventata leonessa, rapace, crudele [...] alli altri animali [...]; è molto lasciva, [...] sta fra li pensieri lionini, pieni di crudeltà, e in quelli si riposa ${ }^{7}$.

Appellandosi poi alla metafora gregoriana «In medio leunculorum enutrivit catulos suos», Savonarola così ne esplicita ulteriormente il senso:

[...] Nel mezzo delli lioncini ha nutrito li suoi figlioli; [...]; Guarda nelle corti de' prelati, che sono nutriti li figliuoli tra' leoncelli. Sono mescolati prelati, preti, con secolari, leoni con lioncelli; [...] et leo factus est [...] Ella ha tolto uno lioncello [...] hallo fatto lione. Guarda colui che cava uno cortigiano pieno di vizii [...] e mettelo in uno beneficio a governare le anime: questo è tôrre un lioncello e farlo leone ${ }^{8}$.

Il primo livello della metafora, dunque, sancisce la mutazione zoomorfa della madre Chiesa da donna a leonessa, assopita tra i leoni e assorta nei suoi 'pensieri leonini' (fatti di rapacità, crudeltà e lascivia); i leoni che l'attorniano ne sono i ministri e reggitori (principi, prelati, preti), sciaguratamente deputati al governo delle anime. Il secondo livello di questa complessa costruzione simbolica vede la leonessa crescere e nutrire i suoi cuccioli tra quelli che il frate chiama «leoncini» o «leoncelli»: è un severo monito mosso alla promiscuità delle «corti dei prelati», ove "son mescolati prelati, preti con secolari, leoni con leoncelli». All'apparenza un innocuo vezzeggiativo, «leoncello» sta a indicare invero una progenie imperfetta e adulterata, che si mescola arbitrariamente con i cuccioli sani, in cui Savonarola adombra gli uomini mondani (altra volta detti «ladroncelli»). L'insano ciclo si compie nel terzo livello della metafora, che vede la leonessa scegliere uno fra i leoncelli per farne un leone: è il «cortigiano pieno di vizi» divenuto tiranno e messo così a governare le anime dall'«intelligenza dei cattivi», che «vorria fare uno leoncello leone, cioè tiranno».

8 Altra pregressa figurazione metaforica del tiranno rintracciabile nel 'bestiario' savonaroliano è quella del nibbio, uccello da preda notoriamente assunto a referente simbolico del vizio dell'invidia già nella Storia Naturale di Plinio il Vecchio e in testi medievali ben conosciuti nella Firenze dello scorcio del secolo. Il 19 dicembre 1494, pochi giorni prima della promulgazione del nuovo ordinamento fiorentino (avvenuta il 23 dicembre), con la predica ${ }^{\circ}$ XIX del ciclo sopra Aggeo il ferrarese così ammoniva $\mathrm{i}$ cittadini all'auspicata istituzione del Consiglio Grande, a riforma dell'ammalorata repubblica e a prevenzione dell'avvento di un tiranno, ormai percepito come imminente: «E se voi farete questo che v'ho detto, beati voi; ma se voi non lo farete, rovinerete voi medesimi e la città; e verrà il nibbio e porterà via el proprio, el commune e piglieravvi tutti»9.

Il rischio che non solo del "proprio», vale a dire del privato patrimonio di ciascuno, ma del (bene) "comune" nella sua interezza il tiranno predatore giungesse a fare incetta, come uccello di rapina, era agli occhi del frate reso pericolosamente verosimile dalla minaccia costituita dalla iniqua condotta di quei cattivi cittadini di estrazione ottimatizia, che, pur di fazione anti-medicea, si opponevano alla pacificazione 
universale propugnata da Savonarola, perché «non amono il bene commune, ma solo pensono alla loro utilità e vorrebbono pigliare questa lepre e appropiarsela a loro» ${ }^{10}$.

È l'11 gennaio del $1495 \mathrm{e}$, nella seconda predica del ciclo sopra i Salmi, a compimento della metafora venatoria, Firenze appare inerme preda leprina, in balia (ancor prima che dell'assalto del nibbio tirannico) delle angherie di «cani stizziti»: «vedevo in fantasia molti cani che seguitavano una lepre; venne uno uomo con una spada in mano e fecesi incontro a questi cani e spaventogli e la lepre fuggì via» ${ }^{11}$.

11 La frustrante stizza subentrata per la fallita razzia nel branco di cani, che «vennono in tanta rabbia che si mordevono la lingua e sputandola nella faccia l'uno all'altro e poi si mordevono e si mangiavono l'un l'altro» ${ }^{12}$, genera quasi incidentalmente il conio della inusitata dicitura di «arrabbiati» (poi di diffusa fortuna nel repertorio savonaroliano), qui per la prima volta impiegata per designare i «cattivi», perciò identificati e marchiati antropologicamente per la loro rabbiosa e vorace ambizione. L'uomo giunto a disperdere i cani armato di spada (evidente riferimento traslato al Gladius Domini), fa a sua volta il paio con Mosè munito di verga (predica ${ }^{\circ}$ XI del 7 marzo 1498, dal ciclo sopra l'Esodo):

Ecco qua la virga di Moisè, con la quale lui dirizzava le pecore: questa dottrina ha

dirizzato le anime nella via del ben vivere. Moisè cacciava e' lupi con quella virga: questa dottrina ha dato di gran bastonate $\mathrm{a}^{\prime}$ lupi ${ }^{13}$.

E, immaginando di rispondere a un'obiezione ( $« \mathrm{O}$ frate, e’ son pastori»), Girolamo aggiungeva: «[...] Non dire così. - Non sono pastori [...] ma sono lupi. Io parlo dei cattivi pastori, non dico de' buoni» ${ }^{14}$.

È ripresa fedele non solo (in via contrastiva) dell'immagine canonica del «buon pastore» ma anche della metafora evangelica dei lupi rapaces (Matteo, 7:15), mediante la quale $i$ «falsi profeti» erano stigmatizzati alla stregua di lupi travestiti da pecore: identificazione, quand'anche fittizia, impossibile e impraticabile per il domenicano, essendo a suo avviso irriducibile l'aporia per cui «non stan lupo e pecora insieme, due contrari non si patiscono» (predica ${ }^{\circ}$ VI del 2 marzo 1498, dal ciclo sopra l'Esodo).

13 È significativo che la perdurante efficacia delle immagini animalistiche della predicazione di Savonarola trovi puntuali rimandi e riscontri, anche oltre la tragica soglia cronologica della morte del frate (23 maggio 1498), nella produzione figurativa di artisti noti per essere stati scossi con veemenza dalle sue severe reprimende morali, al punto di maturare una 'conversione' del proprio stile espressivo, risanandolo dalle pregresse compromissioni con l'estetica gaudente ed edonistica, di matrice secolare e antiquariale, che aveva contraddistinto la stagione medicea. Esemplare, in tal senso, è stata sempre considerata la vicenda del tardo Botticelli: se il manoscritto delle Giornate del savonaroliano Lorenzo Violi (Firenze, Biblioteca Nazionale, Codici Conventi Soppressi, San Marco, J.10.32) contiene riferimenti tanto a Sandro quanto a suo fratello Simone Filipepi, è proprio quest'ultimo a segnalarsi quale autore di una Cronaca, anch'essa manoscritta (Archivio Vaticano, Politicorum XLVII, ff. 338 ss.), che consiste in una sorta di diario politico riferito ai fatti fiorentini accaduti nel decennio 1490-1500, dunque in piena temperie savonaroliana. Questi documenti hanno persuaso gli studiosi botticelliani circa l'attendibilità del racconto vasariano nella vita di Sandro (1550 e 1568): «[...] Della setta del quale [Savonarola] fu in guisa di partigiano, che ciò fu causa che egli abbandonando il dipingere e non avendo entrate da vivere, precipitò in disordine grandissimo». 
Non si accoglie, di quanto dice l'aretino, l'informazione letterale secondo cui Botticelli rinunciasse del tutto alla professione pittorica finendo in ristrettezze e miseria a causa dei mancati guadagni; piuttosto, si è inclini a riconoscere nella fase ultima della sua carriera una soggiacente riflessione, che si attesta come cupamente penitenziale nella sua genesi e persino, in certo qual senso, catartica nel suo epilogo. Botticelli dà prova di una convinta e febbrile adesione ai dettami savonaroliani non solo per la scelta dei temi iconografici - che, abbandonate le estenuate eleganze formali del repertorio mitologico e classicheggiante, che era stato spesso pervaso di compiaciute ancorché non provocatorie cifre sensuali ed erotiche, si volge alla sobria e austera celebrazione dei temi fondamentali del contrito misticismo "piagnone»- ma anche per una peculiare involuzione 'primitivistica' nel suo stesso linguaggio figurativo, ormai ispirato a un intenzionale 'medievalismo' della visione, reso tangibile dal ripudio di quelle stesse essenziali conquiste della proto-modernità artistica dell'Umanesimo di primo Quattrocento: su tutte, il raggiungimento della profondità prospettica, sacrificata e schiacciata nei tardi dipinti botticelliani a favore di una scelta irrealistica di dimensionamento delle figure (proporzionate piuttosto alla loro statura teologica che alla reale collocazione spaziale).

Databile a ridosso dell'ultimo biennio di vita del frate di San Marco (1497-1498) o anche in anni immediatamente successivi alla sua morte sul rogo (1498-1500 circa), la cosiddetta Crocifissione mistica (o simbolica) emerse all'attenzione degli esegeti nel 1924, all'atto della sua entrata nelle collezioni del Fogg Art Museum di Cambridge (Massachusetts) $^{15}$. Originariamente su tavola, fu trasposta su tela l'anno seguente, operazione che ne mise tragicamente a nudo le critiche condizioni di conservazione: la martoriata superficie pittorica, fortemente abrasa e disseminata di cospicue cadute del colore dato a tempera e a olio, concorre con lo stile già di per sé scarno e tormentato della composizione a un risultato di problematica sintesi espressiva, anche dovuto alle sue ridotte dimensioni (appena 72,4 x 51,4 cm). L'opera vanta un'antica provenienza fiorentina da Palazzo Medici Riccardi e transitò nella collezione di Edouard Aynard a Lyon tra il 1900 e il 1913; fu poi di proprietà von Nemes a Monaco sino al 1922 e infine pervenne alla sede odierna tramite Edwar Forbes. La sua consunzione, pur ostacolandone una piena leggibilità, non impedisce di cogliere come alcuni passaggi della sua costruzione iconografica, per unanime parere degli esperti botticelliani, rammentino strettamente, in particolare, la terza predica del ciclo savonaroliano sopra i Salmi, pronunciata il 13 gennaio 1495:

[...] Vedevo per imaginazione una croce nera sopra la Babillonia Roma, nella quale croce era scritto «Ira Domini», e qui sopra pioveva spade, coltelli, lance e ogni arme, e grandine e sassi con tempesta, e fulguri mirabili e grandissime con un tempo oscurissimo e tenebroso. E vedevo un'altra croce d'oro, che agiugneva di cielo insino in terra sopra Jerusalem, nella quale era scritto «Misericordia Dei», e qui era uno tempo sereno, limpidissimo e chiaro; onde, per questa visione, ti dico che la Chiesa di Dio si debbe rinovare e presto, perché Iddio è adirato; e di poi l'infedeli s'hanno a convertire e sarà presto.

Un'altra immaginazione: Vedevo una spada che era sopra Italia e vibrava, e vedevo li Angeli che venivono ed avevono la croce rossa da una mano e dall'altra molte stole bianche [...] ed avevano dall'altra mano el calice pieno ${ }^{16}$.

Le due distinte "visioni» (quelle che il frate indica come immaginazioni) sembrano compendiate in ibridazione nella composizione di Botticelli: il contrasto tra «un tempo oscurissimo e tenebroso» e "un tempo sereno, limpidissimo e chiaro», a significare l'alternasi dell'ira e della misericordia di Dio, prende effettivamente forma nello sfondo 
del dipinto, ove le due metà di cielo separate longitudinalmente dalla croce vedono, a destra, i nembi attorti di buie nubi temporalesche contrapporsi alla luce tersa e all'atmosfera limpida della sezione a sinistra (sovrastata da Dio Padre entro una fulgida mandorla, in luogo della "croce d'oro»); quelle che di primo acchito parrebbero piccole nuvole biache, eteree e rarefatte, si dimostrano a un più ravvicinato sguardo essere soavi figure angeliche, corrispondenti, infatti, agli «angeli che venivono ed avevano la croce rossa da una mano e molte stole bianche» della seconda visione (nel dipinto essi reggono scudi crociati); all'opposto, le armi molteplici («spade, coltelli, lancie»), precipitate come pioggia tra «fulgure mirabili e grandissime», sono sostituite da Sandro con figure demoniache che agitano fiaccole ardenti. L'ambivalente raffronto, di palese ispirazione agostiniana, tra la Babilonia corrotta (identificata con Roma, sede nell'improbo pontificato borgiano) e la Gerusalemme terrena, si ricompone, nell'immagine di Sandro, nell'unica veduta urbana della Firenze contemporanea, con i suoi principali monumenti bene in vista (dal Battistero al Duomo, dalle Cappelle Medicee a Palazzo Vecchio), come a voler alludere al compiersi dell'intero ciclo di 'rinnovazione' entro il perimetro stesso della città del giglio, volta a volta riguardata come Babilonia da redimere e Gerusalemme risorta.

Due figure animalesche accompagnano i protagonisti della sacra rappresentazione che si staglia sul proscenio: a destra, un candido angelo fustigatore è in procinto di vibrare un colpo su una bestia che regge per le zampe posteriori; a sinistra, un'altra fiera, come messa in fuga, sguscia dal manto vermiglio della Maddalena prostrata ad abbracciare la croce. Entrambi gli animali sono rappresentati in attitudine selvaggia e minacciosa, con le fauci spalancate, eppure in condizione di sopraffazione. Nel primo caso, nonostante non siano mancate ipotesi fantasiose (orientate a riconoscervi una donnola o una lontra), il manto fulvo, le zampe feline e l'accenno di una criniera convincono di un'identificazione o, in via generica, con il simbolo civico-politico del Marzocco oppure - secondo una più spiccata aderenza ai dettami savonaroliani - con il tirannico «leoncello leone». L'arma protesa dall'angelo, talvolta intesa come spada, appare di natura flessibile, alla stregua di una sferza, così da potervi scorgere un possibile rimando alla «virga di Moisè». D'altro canto, la fiera che digrignando le zanne sbuca dalla veste della Maddalena, per solito riconosciuta come un lupo rapace (stigma di falso profeta o prelato corrotto), potrebbe anche essere un «cane stizzito» (referente metaforico animalistico, come si è poc'anzi osservato, per la rancorosa fazione dei cattivi cittadini ovvero degli «arrabbiati»).

Più che come una letterale e puntuale trasposizione ecfrastica di un unico sermone savonaroliano, il dipinto botticelliano, dunque, pare offrirsi quale variegato palinsesto figurativo, in cui la personale sensibilità dell'artista, in una bruciante manifestazione delle vive e perduranti suggestioni del retaggio del ferrarese operanti sul suo immaginario, rivisita e ricompone in un dispositivo iconografico del tutto originale elementi simbolici attinti a luoghi e momenti disparati del misticismo fratesco: la possibilità di leggere, nelle due sconfitte bestie feroci, i riferimenti metaforici a due specifici attori politici, di peculiare invenzione savonaroliana (il «leoncello leone» come Marzocco degenerato, a significare il tiranno che i cattivi cittadini vorrebbero insediare; un «cane stizzito», ad adombrare l'atteggiamento rabbioso di questi ultimi), entro uno scenario dai connotati apocalittici caratterizzato da una riconoscibile veduta di Firenze (in asse con la rappresentazione in primo piano della Maddalena, a volerne personificare l'atto di penitenza e devozione a Cristo), corrobora il convincimento 
secondo cui, oltre che dai contenuti eminentemente morali della predicazione di Savonarola, Sandro sia rimasto impressionato dalle icastiche figurazioni politiche (anche e specialmente di tipo animalistico) dal frate messe in campo a Firenze nell'ultimo lustro del Quattrocento.

\section{BIBLIOGRAFIA}

ACIDINI LuCHINAT Cristina, Botticelli. Allegorie mitologiche, Milano, Electa, 2001.

Borelli Anna e PASTORE PASSARo Maria (a cura di), Selected Writings of Girolamo Savonarola: Religion and Politics, 1490-1498, New Haven-London, Yale University Press, 2006.

CAPRETTI Elena, Botticelli, Firenze, Giunti, 1997.

CECCHI Alessandro, Botticelli e l'età di Lorenzo il Magnifico, Firenze, E-ducation Scala Group, 2007.

CECCHI Alessandro e SPIKE John, Botticelli and the Search for the Divine: Florentine Paintings between the Medici and the Bonfires of the Vanities, Boston, Museum of Fine Arts, 2017.

CoRnINI Guido, Botticelli («Art dossier», n 49), Firenze, Giunti, 1990 (rist. 2015).

DeIMLING Barbara, Botticelli, Köln, Taschen, 1994 (rist. 2004).

DE MAIO Romeo, Riforme e miti nella Chiesa del Cinquecento, Napoli, Guida, 1992.

EDELHEIT Amos, Ficino, Pico and Savonarola. The Evolution of Humanist Theology, 1461/2-1498, LeidenBoston, Brill, 2008.

HALL Marcia B., Savonarola's Preaching and the Patronage of Art, in T. Verdon e J. Henderson (a cura di), Christianity and the Renaissance: Image and Religious Imagination in the Quattrocento, Syracuse (NY), Syracuse University Press, 1990.

LightBown Ronald W., Sandro Botticelli. Life and Work, Berkeley and Los Angeles, University of California Press, 1978.

MCQueEn Alison, Political Realism in Apocalyptic Times, Cambridge, Cambridge University Press, 2017.

Malaguzz Silvia, Botticelli. L'artista e le opere, Firenze, Giunti, 2003.

MARIETTI Marina, Emploi des figures du discours dans la prédication de Savonarole, in A. Fontes, J.-L. Fournel e M. Plaisance (dir.), Savonarole : enjeux, débats, questions, Paris, Centre interuniversitaire de recherche sur la Renaissance italienne, Université Sorbonne Nouvelle, 1997, pp. 1-17.

MARIETTI Marina, La figura del tiranno nella predicazione di Girolamo Savonarola, «Chroniques italiennes», série web, $\mathrm{n}^{\circ}$ 11, 2007, pp. 1-27.

Pons Nicoletta, Botticelli. Catalogo completo, Milano, Rizzoli, 1989.

Rowland Christopher, Radical Prophet. The Mystics, Subversives and Visionaries who Strove for Heaven on Earth, London-New York, I. B. Tauris \& Co., 2017. 
SAVonARola Girolamo, Prediche sopra l'Esodo, a cura di P. G. Ricci, 2 voll., Roma, Angelo Belardetti, «Edizione nazionale delle opere», 1955-1956.

SAVONARola Girolamo, Prediche sopra Ezechiele, a cura di R. Ridolfi, 2 voll., Roma, Angelo Belardetti, «Edizione nazionale delle opere», 1955.

SAVonARola Girolamo, Prediche sopra Giobbe, a cura di R. Ridolfi, 2 voll., Roma, Angelo Belardetti, «Edizione nazionale delle opere», 1957.

SAVONAROLA Girolamo, Prediche sopra Aggeo; con il Trattato circa il reggimento e governo della città di Firenze, a cura di L. Firpo, Roma, Angelo Belardetti, «Edizione nazionale delle opere», 1965.

SAVONAROLA Girolamo, Prediche sopra i Salmi, a cura di V. Romano, 2 voll., Roma, Angelo Belardetti, «Edizione nazionale delle opere», 1969-1974.

Savonarola Girolamo, Prediche sopra Amos e Zaccaria, a cura di P. Ghiglieri, 3 voll., Roma, Angelo Belardetti, «Edizione nazionale delle opere», 1971-1972.

STEINBERG Ronald M., Fra Girolamo Savonarola, Florentine Art and Renaissance Historiography, Athens (OH), Ohio University Press, 1977.

TERREAUX-SCOTTO Cécile, « Mon dire est un faire ». L'art de persuader dans les sermons politiques de Savonarole, «Cahiers d'études italiennes», $\mathrm{n}^{\circ}$ 2, 2005, pp. 89-117.

Venturelli Piero, Per una città umana. Savonarola ovvero Mosé a Firenze, in T. Casadei (a cura di), Esodo, Santarcangelo di Romagna (RN), Fara, 1999, pp. 78-98.

VERESS Ferenc, Note per un problema storiografico: Savonarola e le arti, in A. Tüskès (a cura di), Ars Perennis, Budapest, CentrArt, 2009, pp. 261-265.

VILADESAu Richard, The Triumph of the Cross. The Passion of Christ in Theology and the Arts, from the Renaissance to the Counter-Reformation, Oxford, Oxford University Press, 2008.

VITI Paolo, Savonarola e la tradizione repubblicana fiorentina, in G. C. Garfagnini (a cura di), Savonarola. Democrazia, Tirannide, Profezia, Firenze, Sismel-Edizioni del Galluzzo, 1998, pp. 55-65.

\section{NOTE}

1. G. Savonarola, Prediche sopra Amos e Zaccaria, a cura di P. Ghiglieri, 3 voll., Roma, Angelo Belardetti, 1971, predica VIII, vol. 1, p. 233 [24 febbraio 1496].

2. Id., Prediche sopra l'Esodo, a cura di P. G. Ricci, 2 voll., Roma, Angelo Belardetti, 1955, predica VII, vol. 1, p. 179.

3. Id., Prediche sopra Giobbe, a cura di R. Ridolfi, 2 voll., Roma, Angelo Belardetti, 1957, predica XXVI, vol. 2, p. 29 [1495].

4. Id., Prediche sopra Amos, cit., predica IX, vol. 1, p. 242.

5. Id., Prediche sopra $i$ Salmi, a cura di V. Romano, 2 voll., Roma, Angelo Belardetti, 1974, predica XXIV, vol. 2, p. 117.

6. Id., Prediche sopra Ezechiele, a cura di R. Ridolfi, 2 voll., Roma, Angelo Belardetti, predica LIII, vol. 2, p. 359.

7. Ivi, predica XL, vol. 2, pp. 204-205.

8. Ivi, pp. 206-207.

9. Id., Prediche sopra Aggeo con il Trattato circa il reggimento e governo della città di Firenze, a cura di

L. Firpo, Roma, Angelo Belardetti, 1965, predica XIX, p. 337.

10. Id., Prediche sopra i Salmi, cit., predica II, vol. 1, p. 26. 


\author{
11. Ibid. \\ 12. Ibid. \\ 13. G. Savonarola, Prediche sopra l'Esodo, cit., predica XI, vol. 1, p. 313. \\ 14. Ibid. \\ 15. Vedi <www.harvardartmuseums.org/art/299923>. \\ 16. G. Savonarola, Prediche sopra i Salmi, cit., predica III, vol. 1, p. 52.
}

\title{
RIASSUNTI
}

L'obiettivo del presente contributo è di interpretare un corpus di sermoni pronunciati da Savonarola a Firenze a più riprese, principalmente nel periodo tra il 1495 e il 1497. Il filo conduttore è rappresentato dalle metafore animalistiche dietro le quali il frate cela i principali attori della contemporanea ribalta politica fiorentina: dai «cani stizziti» come simbolo della fazione degli «arrabbiati» (i cattivi cittadini, vale a dire gli ottimati avversi all'oligarchia medicea) alla stessa cittadinanza fiorentina figurata come preda in guisa di lepre (secondo sermone del ciclo sui Salmi, 11 gennaio 1495), sino alla metafora della Chiesa come leonessa che accudisce i propri cuccioli (nei quali sono adombrati preti e prelati corrotti), tra i quali per istigazione dei cattivi cittadini potrebbe essere elevato a tiranno un «leoncello leone» (sermone XL del ciclo su Ezechiele, 12 marzo 1497). La parte finale del contributo è dedicata a un'analisi iconografica della Crocifissione mistica di Botticelli (Cambridge, Mass., Fogg Art Museum), dipinta tra il 1498 e il 1500 o subito dopo, sotto la viva influenza della predicazione savonaroliana, con speciale riguardo alla presenza di figure animali: il piccolo leone fustigato dall'angelo e il canide (un cane rabbioso o un lupo rapace) che sguscia dal manto della Maddalena penitente.

Cette contribution vise à interpréter une série de sermons prêchés par Savonarole à Florence, à plusieurs reprises, notamment entre 1495 et 1497. Le fil rouge du discours sera représenté par les images d'animaux derrière lesquelles le frère a caché des acteurs fondamentaux du scénario politique florentin à la fin du xv siècle : les «cani stizziti » («chiens irrités ») qui symbolisent la faction des «arrabbiati» (les colériques, appelés aussi «cattivi», " mauvais », c'est-à-dire les «ottimati » opposés aux oligarques fidèles aux Médicis ; les citoyens florentins figurés en tant que proie sous la forme d'un « lièvre » dans le deuxième sermon du cycle sur les Psaumes prononcé le 11 janvier 1495 ; enfin la métaphore de l'Église-lionne, «leonessa », qui prend soin de ses propres lionceaux, «leoncelli »-qui représentent les prêtres et les prélats corrompus. Parmi ceux-ci, un «leoncello leone» («jeune lion») pourrait prendre la place du tyran à l'instigation des «cattivi » (sermon XL du cycle sur Ézéchiel, 12 mars 1497). La partie finale est consacrée à la lecture iconographique de la Crucifixion mystique peinte par Botticelli pendant les années 1498-1500 (aujourd'hui au Fogg Art Museum de Cambridge, Massachusetts), dont la signification est inspirée par la prédication de Savonarole, à travers la présence d'animaux symboliques : le lion, bien sûr, mais aussi le loup de la métaphore évangélique (Matthieu, 7:15), reprise par Savonarole dès 1493 pour évoquer la corruption cléricale.

The present contribution aims to interpret a series of sermons pronounced by Savonarola in Florence on several occasions, mainly between 1495 and 1497. The common denominator is represented by the animal images behind which the friar hides the pivotal actors of the 
contemporary political limelight in late 15th century Florence: from the "cani stizziti" (rabid dogs) as symbols of the faction of the "arrabbiati" (the angry and irascible citizens, i.e. the optimates averse to the Medicean oligarchy) to the Florentine community figured like a hunting prey in the guise of a hare (second sermon of the cycle on the Psalms, 11 January 1495), until the metaphor of the Church as a lioness, with her puppies ("leoncelli") overshadowing the corrupted priests and prelates, among which a "leoncello leone" would be taken as a new tyrant, because of the bad citizens' instigation (sermon XL of the cycle on Ezekiel, 12 March 1497). The final part of the paper is devoted to an iconographic analysis of Botticelli's Mystical Crucifixion (Cambridge, Mass., Fogg Art Museum), painted around 1498-1500 or shortly later, under the decisive influence of Savonarola's preaching, with particular reference to the animal figures present in it: the lion scourged by the angel and the canid (a rabid dog or vicious wolf) emerging from the penitent Magdalene's drapery.

INDICE

Mots-clés : Savonarole, Botticelli, Florence, métaphore, allégorie

Parole chiave : Savonarola, Botticelli, Firenze, metafora, allegoria

Keywords : Savonarola, Botticelli, Florence, metaphor, allegory

\section{AUTORE \\ MARCO VERSIERO \\ Chercheur indépendant. \\ versiero@yahoo.it}

